\title{
ACOMPANHAMENTO FARMACOTERAPÊUTICO EM UNIDADE BÁSICA DE SAÚDE
}

\author{
PHARMACOTHERAPEUTIC FOLLOW-UP IN A BASIC HEALTH UNIT
}

\author{
Anna Júlia de Souza Freitas ${ }^{1}$ \\ Dayverson Luan de Araújo Guimarães ${ }^{2}$ \\ Maria Fátima Gonçalves de Araújo ${ }^{3}$ \\ Maria Luisa de Sá Vieira \\ Monalisa Ferreira de Lucena ${ }^{5}$ \\ Maria do Socorro Ramos de Queiroz ${ }^{6}$
}

RESUMO: OBJETIVO: Identificar os Resultados Negativos à Medicamentos (RNMs) em pacientes acompanhados em uma Estratégia de Saúde da Família do município de Campina Grande-PB. METODOLOGIA: O estudo foi do tipo transversal, com abordagem quantitativa e descritiva. $O$ instrumento utilizado para coleta de dados do perfil farmacoterapêutico foi o preconizado pelo Manual de Cuidados Farmacêuticos, elaborado pelo Ministério da Saúde. Os participantes foram abordados sem a presença de terceiros, em ambiente fechado. RESULTADOS: O grupo de usuários foi representado por 49 pacientes $(72 \%)$ portadores de Hipertensão Arterial Sistêmica (HAS), 18 pacientes (26\%) portadores de HAS e Diabetes Mellitus tipo 2 (DM2) e apenas 1 paciente $(2 \%)$ era portador apenas de DM2. O papel do farmacêutico na atenção aos idosos é o monitoramento da prescrição, a garantia da efetividade e segurança da farmacoterapia, tendo como objetivo principal minimizar impactos sobre a saúde desses indivíduos. Identificou-se nesse trabalho a prevalência dos RNM's e assim pode- se intervir nestes casos, possibilitando a resolução do problema através da interação com a equipe multidisciplinar em saúde. CONCLUSÃO: Portanto, é de fundamental importância o farmacêutico

\footnotetext{
1 Acadêmica do curso de Farmácia da Universidade Estadual da Paraíba - UEPB. E-mail: ajsfreitas22@gmai.com.

${ }_{2}^{2}$ Acadêmico do curso de Farmácia da Universidade Estadual da Paraíba - UEPB. E-mail: dayversonluan@hotmail.com.

3 Acadêmica do curso de Farmácia da Universidade Estadual da Paraíba - UEPB. E-mail: fattaraujo27@gmail.com.

${ }^{4}$ Mestranda do Programa de Pós-Graduação em Saúde Pública da Universidade Estadual da Paraíba - UEPB. E- mail: marialuisasavieira@gmail.com.

${ }^{5}$ Mestranda do Programa de Pós-Graduação em Ciências Farmacêuticas da Universidade Estadual da Paraíba - UEPB. E-mail: Monalisa.lucena16@gmail.com.

6 Professora do Curso de Farmácia da Universidade Estadual da Paraíba - UEPB. E-mail: queirozsocorroramos@yahoo.com.br.
} 
inserido na equipe multidisciplinar em saúde, pois ele é capaz de realizar a monitorização terapêutica dos medicamentos, revisão da farmacoterapia e acompanhamento farmacoterapêutico.

Palavras chave: Hipertensão arterial; Diabetes mellitus; Revisão da farmacoterapia.

ABSTRACT: OBJECTIVE: To identify the Negative Results to Medicines (MRIs) in patients followed up in a Family Health Strategy in the city of Campina Grande-PB. MATERIALS AND METHODS: The study was cross-sectional, with a quantitative and descriptive approach. The instrument used to collect data on the pharmacotherapeutic profile was the one recommended by the Pharmaceutical Care Manual, prepared by the Brazil Health Ministry. The participants were approached without the presence of third parties, in a closed environment. RESULTS: The user group was represented by 49 patients (72\%) with Systemic Arterial Hypertension (SAH), 18 patients (26\%) with SAH and Type 2 Diabetes Mellitus (DM2) and only 1 patient (2\%) was bearer of DM2 only. The pharmacist's role in caring for the elderly is monitoring the prescription, ensuring the effectiveness and safety of pharmacotherapy, with the main objective of minimizing impacts on the health of these individuals. In this work, the prevalence of MRIs was identified and thus it is possible to intervene in these cases, enabling the problem to be solved through interaction with the multidisciplinary health team. CONCLUSION: Therefore, the Pharmacist inserted in the multidisciplinary health team is of fundamental importance, as he is able to perform the therapeutic monitoring of drugs, review of pharmacotherapy and pharmacotherapeutic monitoring.

Keywords: Arterial Hypertension; Diabetes mellitus; Pharmacotherapy revision. 


\section{INTRODUÇÃO}

Um modelo da prática clínica na Atenção Básica de Saúde (ABS) ocorre através do cuidado farmacêutico ao usuário que é composto por quatro etapas: a coleta e organização dos dados do usuário; a avaliação e identificação de problemas relacionados à farmacoterapia; a pactuação de um plano de cuidado com o usuário e o seguimento individual do usuário quando necessário (WONG, 2004; CORRER; OTUKI, 2013).

O cuidado farmacêutico constitui um conjunto de ações e serviços desenvolvidos no Sistema Único de Saúde que inclui a educação em saúde para a equipe e usuário, atividades de promoção da saúde de caráter geral e ações de promoção do uso racional de medicamentos, com o desenvolvimento de atividades clínico-assistenciais e técnico-pedagógicas. A atividade assistencial, praticada nos pontos de atenção, inclui os serviços de clínica farmacêutica, que podem ser ofertados ao usuário de forma individual e/ou em atendimentos compartilhados com outros membros da equipe de saúde. As atividades técnico-pedagógicas, de forma complementar, visam à educação e a conscientização da equipe de saúde e da comunidade para a promoção do uso racional de medicamentos (BRASIL, 2015).

No cuidado farmacêutico realiza-se a avaliação da história farmacoterapêutica do paciente, a qual é obtida em dois passos, primeiramente, com auxílio de uma tabela, é registrada a farmacoterapia atual do paciente, ou seja, os medicamentos que o paciente está fazendo uso no momento, posteriormente, é avaliada a adesão do paciente ao tratamento, como também a sua capacidade de gerir os seus medicamentos, reações adversas, alguma dificuldade para usar os medicamentos, o acesso a esses, às alergias e às terapias alternativas (WONG, 2004; CORRER; OTUKI, 2013; CRF, 2017).

É importante saber que no Brasil e no mundo as doenças crônicas não transmissíveis como Hipertensão Arterial Sistêmica (HAS) e de Diabetes Mellitus (DM), são de alta prevalência e os portadores destes agravos são usuários de 
medicamentos de uso contínuo, muitas vezes utilizam dois ou mais itens sendo mais suscetíveis ao seu uso inadequado, à polifarmácia e às interações medicamentosas (PRADO; FRANCISCO; BARROS, 2016; SILVA, et al., 2016).

Esses pacientes precisam ter uma boa relação e confiança no farmacêutico para que seja desenvolvido um trabalho conjunto que vise sempre a melhora na saúde, sabido que o tratamento dessas doenças crônicas é contínuo, e o bem-estar do paciente está diretamente ligado ao controle das mesmas, que só é possível com o uso correto dos medicamentos.

Considerando a alta incidência de HAS e DM na população, a importância do medicamento no tratamento e controle de tais patologias e o papel fundamental do farmacêutico no uso racional desses medicamentos, o presente trabalho teve por objetivo identificar os Resultados Negativos à Medicamentos (RNMs) em pacientes acompanhados em uma Estratégia de Saúde da Família do município de Campina Grande-PB.

\section{MATERIAL E MÉTODOS}

O estudo foi do tipo transversal, com abordagem quantitativa e descritiva, realizado no período de janeiro a abril de 2019 e focado na avaliação do perfil farmacoterapêutico de usuários hipertensos e/ou diabéticos domiciliados cadastrados na Unidade Básica de Saúde da Família Bonald Filho, em Campina Grande-PB. A pesquisa foi autorizada pelo comitê de ética em pesquisa do Centro de Ensino Superior e Desenvolvimento (CESED/PB), em Campina Grande-PB, com parecer de $\mathrm{n}^{\circ}$ 03216818.0.0000.5175 e foi realizado de acordo com os padrões éticos exigidos na resolução $N^{\circ} 466$ de 12 de dezembro de 2012 do Conselho Nacional de Saúde.

O instrumento utilizado para coleta de dados do perfil farmacoterapêutico foi preconizado pelo Manual de Cuidados Farmacêuticos, elaborado pelo Ministério da Saúde (BRASIL, 2015). Os participantes foram abordados sem a presença de terceiros, em ambiente fechado. 
Para avaliar o perfil farmacoterapêutico foi utilizado o Terceiro Consenso de Granada (COMITÊ DE CONSENSO, 2007).

Os resultados foram analisados pelo programa Statistical Package for the Social Sciences (SPSS versão 17.0).

\section{RESULTADOS E DISCUSSÃO}

Participaram da pesquisa 68 pacientes, dos quais 44 (65\%) deles estavam representados pelo gênero feminino. Várias pesquisas realizadas por todo o país detectam a maior procura das mulheres aos serviços de saúde. Estudo semelhante na cidade de Dourados- MS revelou que 64,9\% dos participantes da pesquisa eram do gênero feminino, indicando que as mulheres procuram mais as Unidades de Saúde, obtendo assim resultados mais efetivos no tratamento, bem como tem melhor adesão a terapia farmacológica e não farmacológica (BEZERRA, 2009; CARVALHO; ALMEIDA; GARBINATO, 2012).

A média de idade dos pacientes foi de $63 \pm 13$ anos, dos quais 39 (58\%) deles estavam na faixa etária entre 60 a 74 anos, $11(16 \%)$ na faixa de 75 a 90 anos e 18 pacientes (26\%) se encontraram com 29 a 57 anos de idade.

O grupo de usuários foi representado por 49 pacientes (72\%) portadores de Hipertensão Arterial Sistêmica (HAS), 18 pacientes (26\%) portadores de HAS e Diabetes Mellitus tipo $2(\mathrm{DM} 2)$ e apenas 1 paciente $(2 \%)$ era portador apenas de DM2. A HAS e o DM são dois exemplos importantes de doenças crônico degenerativas, com alta prevalência, alto custo para a sociedade e elevado número de morte da população brasileira. Apresentam aspectos comuns como etiopatogenia, cronicidade e necessidade de controle permanente e fatores de risco semelhantes, tais como, obesidade, dislipidemia e sedentarismo. O tratamento propõe mudanças de vida e nos hábitos alimentares, e é necessário o acompanhamento de uma equipe multidisciplinar, para melhoria do tratamento controle das duas doenças (FELIPETTI et al., 2016). 
De acordo com as classes farmacológicas a mais prescrita no presente estudo foram os diuréticos (48\%), representado pela hidroclorotiazida, seguido dos receptores de angiotensina II (37\%) tendo a losartana como principal fármaco e o hipoglicemiante oral (31\%), a biguanida sendo prescrita a metformina de $850 \mathrm{mg}$.

FIGURA 1 - Classes farmacológicas identificadas no delineamento do perfil farmacoterapêutico.

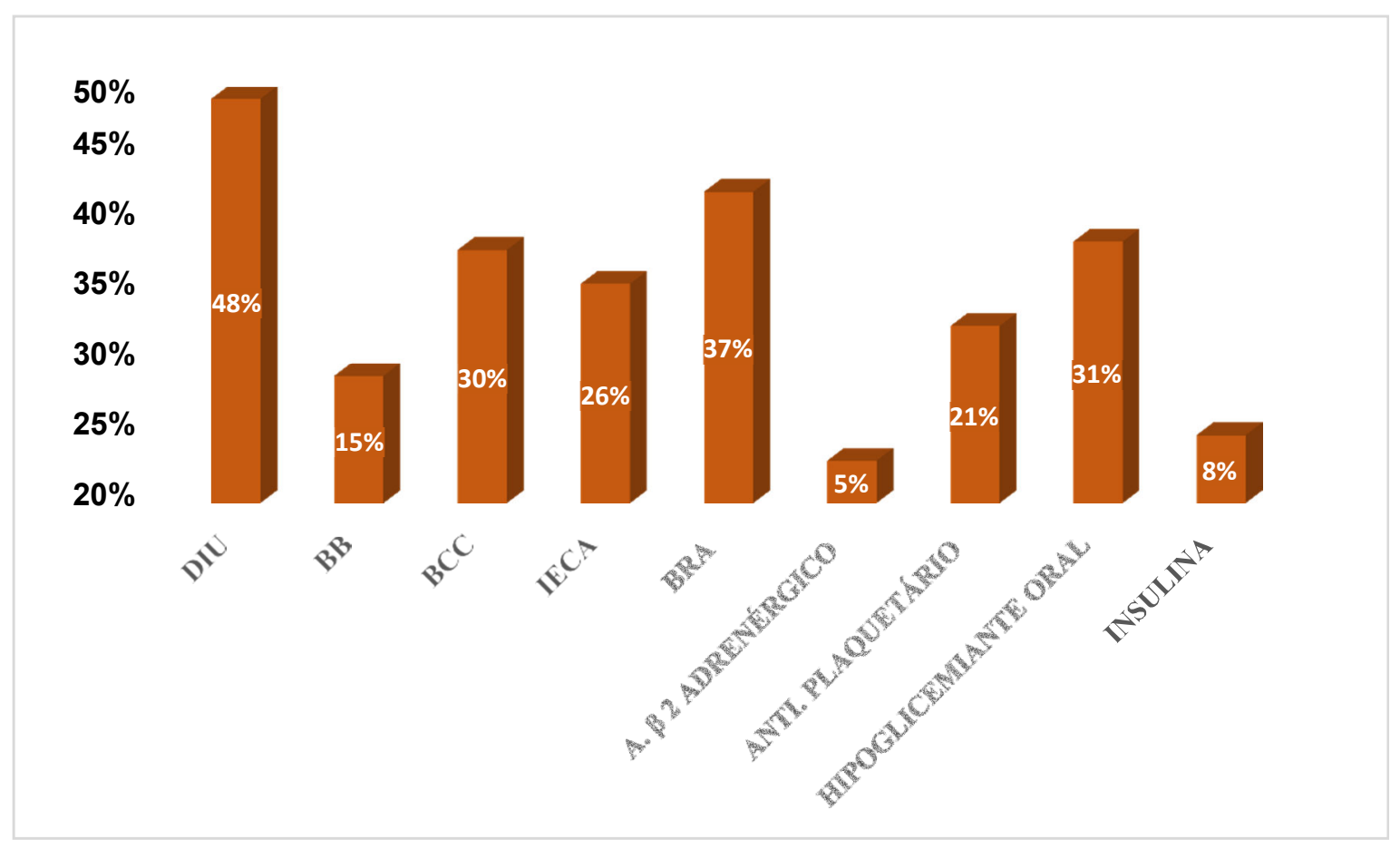

Fonte: Dados da pesquisa.

DIU $=$ Diuréticos $; \mathbf{B B}=$ Betabloqueador $; \mathbf{B C C}=$ Bloqueador dos canais de cálcio; IECA = Inibidores da enzima conversora de angiotensina; $\mathbf{B R A}=$ Bloqueador dos receptores de angiotensina II; A. $\boldsymbol{\beta} 2$ ADRENÉRGICO = Agonista beta-2adrenérgico; ANTI. PLAQUETÁRIO = Antiagregante plaquetário.

Com relação aos tipos de anti-hipertensivos e antidiabéticos utilizados pelos pacientes, esses fármacos citados são fornecidos pelo SUS, integram a Relação Nacional de Medicamentos Essenciais (RENAME) e são distribuídos no grupo Hiperdia. 
A polifarmácia que corresponde o uso regular de cinco ou mais medicamentos, foi identificada na prescrição de alguns pacientes acompanhados. Esta pode ser definida como o uso de múltiplos medicamentos por um paciente. Segundo Clyne et al., (2015) a polifarmácia e a prescrição de medicamentos potencialmente inapropriados para idosos são problemas de saúde pública e têm sido associados com eventos adversos tais como quedas, hospitalização e morte. É um fator preocupante, porque as mudanças fisiológicas relacionadas ao envelhecimento podem alterar as propriedades farmacocinéticas e farmacodinâmicas no paciente idoso (COELHO; MARCOPITO; CASTELO, 2004).

O uso concomitante de múltiplos medicamentos pode trazer diversos desfechos indesejáveis à saúde como o aumento na ocorrência de reações adversas e interações medicamentosas, menor adesão à terapia medicamentosa, diminuição da capacidade funcional e declínio cognitivo do idoso. Além desses prejuízos, há maiores demandas por cuidados assistenciais, elevação do número de admissões hospitalares e dos custos para o sistema de saúde. A polifarmácia também pode afetar a qualidade do tratamento medicamentoso prescrito, quando associada à automedicação, bastante comum entre idosos.

Alguns fatores têm sido associados à polifarmácia entre idosos, como pertencer ao gênero feminino, ter autopercepção de saúde ruim, ser de faixa etária mais avançada, ter baixa escolaridade e referência à presença de doenças crônicas (FELIPETTI ET AL., 2016).

$\mathrm{Na}$ análise de Resultados Relacionados aos Medicamentos (RNM's), constatou-se que 28 pacientes (41\%) apresentavam algum dos tipos, sendo que 20 $(30 \%)$ desses pacientes tinham problemas de Inefetividade não-quantitativa, devido à presença de Interações entre medicamentos, em sua grande maioria. As interações mais frequentes ocorreram entre hidroclorotiazida e glibenclamida. Os resultados referentes a Interações entre medicamentos ocorrem, em geral, quando o efeito de um fármaco é modificado pela presença de outro, aumentando ou diminuindo a eficácia terapêutica, ou até mesmo gerando toxicidade ao organismo. Os casos identificados referem-se ao hipoglicemiante glibenclamida que pode ter sua efetividade reduzida em função do uso do diurético tiazídico. Ainda foi frequente a interação entre o AAS e hidroclorotiazida, em que o ácido pode reduzir a eficácia 
do diurético, muitas vezes indispensável ao tratamento anti-hipertensivo (SANTOS; TORRIANI; BARROS, 2013).

Ainda houve uma parcela de 7 pacientes (10\%) que apresentou RNM's de Segurança. Os casos de Segurança identificados estavam relacionados ao efeito colateral apresentado pelos usuários que é a "tosse seca", eles utilizavam o Inibidor da Enzima Conversora de Angiotensina II (IECA), o captopril. A tosse indesejada levou a realizar a troca desse medicamento por Antagonistas do Receptor de Angiotensina II, que não apresentam estes efeitos indesejados.

Por fim, apenas 1 paciente apresentou RNM de Necessidade, demonstrando níveis pressóricos alterados persistentes e este não fazia uso de alguns dos itens da terapia anti- hipertensiva prescrita. Quanto a ocorrência do RNM referente à Necessidade aconteceu porque os medicamentos que eram prescritos não eram adquiridos muitas vezes por situação financeira, contribuindo assim para o aumento dos níveis pressóricos podendo resultar em um evento cardiovascular.

Durante o processo de envelhecimento, a busca por medicamentos aumenta e o cuidado farmacêutico se torna essencial, para que haja intervenção por meio de orientação e ações educativas que possam proporcionar uma maior qualidade de vida a este paciente. "O papel do farmacêutico na atenção aos idosos é o monitoramento da prescrição, a garantia da efetividade e segurança da farmacoterapia, tendo como objetivo principal minimizar impactos sobre a saúde desses indivíduos" (BALDONI et al., 2014; CARVALHO; SENA, 2017).

\section{CONCLUSÃO}

Foi possível observar também, a prevalência dos RNM's e assim poder intervir nestes casos e possibilitar a resolução do problema através da interação com a equipe multidisciplinar em saúde. Percebe-se neste caso, que é de fundamental importância que o profissional Farmacêutico faça parte da equipe multidisciplinar em Saúde, pois ele é capaz de realizar a monitorização terapêutica dos medicamentos, revisão da farmacoterapia e acompanhamento farmacoterapêutico de cada um 
desses pacientes, a fim de que seja possível estabilizar o quadro da doença crônica não transmissível e dar autonomia para que o paciente se mantenha saudável e tenha controle nas condições de sua saúde, através da educação em saúde.

\section{REFERÊNCIAS BIBLIOGRÁFICAS}

BALDONI, A. O.; DEWULF, N. de L. S.; SANTOS, V. dos; REIS, T. M. dos.; AYRES, L. R.; PEREIRA, L. R. L. Dificuldades de acesso aos serviços farmacêuticos pelos idosos. Rev Ciências Farm Básica e Aplicada. v. 35, n. 4, 2014, p. 615-621. Disponível em: <http://serbib.fcfar.unesp.br/seer/index.php/Cien_Farm/article/viewFile/3224/3224>. Acesso em: 26 nov de 2017.

BEZERRA, D. S.; CARVALHO, D. B.; SILVA, A. S. Avaliação das características dos usuários com hipertensão arterial e/ou diabetes mellitus em uma Unidade de Saúde Pública no município de Jaboatão dos Guararapes - PE. Rev Ciências Farm Básica Aplicada. v. 30, n.1, p. 69- 73, 2009.

BRASIL, Ministério da Saúde. Cuidado farmacêutico na atenção básica: Capacitação para implantação dos serviços de clínica farmacêutica. Brasília: Ministério da Saúde, caderno $n$. 2, 1ed. 308p, 2015.

CARVALHO, M. C.; ALMEIDA, A. P. M.; GARBINATO, L. R. A assistência farmacêutica no atendimento aos pacientes do HIPERDIA da ESF 18 e 19 da cidade de Dourados/MS. Interbio, [S.I.], v. 6, n. 2, p.5-15, 2012.

CARVALHO, J. C.; SENA, C. F. A. Problemas relacionados à manutenção do tratamento medicamentoso em pacientes idosos e as contribuições da atenção farmacêutica. Rev Brasileira de Ciências da Vida, [S.I.], v. 5, n. 1, jul. 2017. ISSN 2525-359X. Disponível em: <http://jornal.faculdadecienciasdavida.com.br/index.php/RBCV/article/view/112>. Acesso em: 26 nov de 2019.

CLYNE, B.; SMITH, S. M.; HUGHES, C. M.; BOLAND, F.; BRADLEY, M. C.; COOPER, J. A.; FAHEY, T. Eficácia de uma intervenção multifacetada para prescrição potencialmente inadequada em pacientes mais velhos na atenção primária: um estudo controlado por randomização de grupo (Estudo OPTI-SCRIPT). Ann Fam Med. v. 13, p. 545-553, 2015.

COELHO, F. J. M.; MARCOPITO, L. F.; CASTELO, A. Perfil de utilização de medicamentos por idosos em área urbana do Nordeste do Brasil. Rev Saúde Pública. v. 38, n. 4, p. 557-564, 2004.

COMITÊ DE CONSENSO. Terceiro consenso de Granada sobre Problemas Relacionados com Medicamentos e Resposta Negativa Associada a Medicamentos. Ars Pharm. Granada. n 1, v. 48, p. 5-12, 2007.

CORRER, C. J.; OTUKI, M. F. A prática farmacêutica na farmácia comunitária. Porto Alegre: Artmed. 2013. 454p.

FELIPETTI, F. A.; HOSHI, A. T.; NASSAR, C. A.; NASSAR, P. O. Prevalência de hipertensos e diabéticos cadastrados e acompanhados pelas unidades de saúde do município de Cascavel Paraná. Rev APS. v. 19, n. 1, p. 77-84, 2016.

GARCIA, L. P.; MAGALHÃES, L. C. G.; SANT'ANNA, A. C.; FREITAS, L.R.S.; AUREA A. P.; 
POSENATO, L. Dimensões do Acesso a Medicamentos no Brasil: Perfil e Desigualdades dos Gastos das Famílias, segundo as Pesquisas de Orçamentos Familiares 2002-2003 e 20082009. Texto para discussão / Instituto de Pesquisa Econômica Aplicada (Ipea). Rio de Janeiro: Ipea, junho de 2013.

PRADO, M. A. M. B.; FRANCISCO, P. M. S. B.; BARROS, M. B. A. Diabetes em idosos: uso de medicamentos e risco de interação medicamentosa. Ciência \& Saúde Coletiva. [S.1], v. 21, n. 11, p. 3447-3458, 2016.

SANTOS, L.; TORRIANI, M. S.; BARROS, E. Medicamentos na prática da farmácia clínica. Porto Alegre: Artmed, 2013. 1120 p.

SILVA, E. C., MARTINS, M. S. A. S., MARTINS, GUIMARÃES, L. V., SEGRI, N. J., LOPES, M. A. L., ESPINOSA, M. M. Prevalência de hipertensão arterial sistêmica e fatores associados em homens e mulheres residentes em municípios da Amazônia Legal. Rev Bras de Epid. [S. 1], v. 19 , n. 1 , p. $38-51,2016$.

WONG, I. C. K. Randomised controlled trials (RCTs) to evaluate complex healthcare interventions: a case study. Pharn World \& Sci. v. 26, n.5, p. 247-252, 2004. 